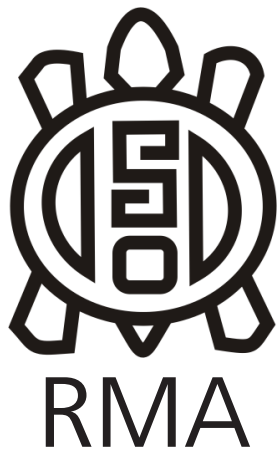

Arqueología

\title{
Primera aproximación al consumo de fauna y al manejo de rebaños en el sur de Pozuelos (Puna de Jujuy, Argentina) en tiempos prehispánicos tardíos y coloniales (siglos XIII a XVIII)
}

First approach to the consumption of fauna and herd management in the south of Pozuelos (Puna de Jujuy, Argentina) in late pre-Hispanic and colonial times (13th to 18th centuries)

Lucrecia M.R. Torres Vega*, Carlos I. Angiorama** y Pablo H. Mercolli***

*Instituto de Arqueología y Museo, Facultad de Ciencias Naturales e I.M.L., Universidad Nacional de Tucumán. Instituto Superior de Estudios Sociales, CONICET. E-mail: Itorresvega@gmail.com

** Instituto de Arqueología y Museo, Facultad de Ciencias Naturales e I.M.L., Universidad Nacional de Tucumán. Instituto Superior de Estudios Sociales, CONICET. E-mail: carlosangiorama@gmail.com *** Instituto Interdisciplinario de Tilcara, UBA. E-mail: pmercolli@hotmail.com

\begin{abstract}
Resumen
El análisis comparativo de distintos conjuntos zooarqueológicos recuperados en sitios del sur de la cuenca de Pozuelos (puna de Jujuy, Argentina), con fechados que los ubican entre los siglos XIII y XVIII, nos permite reconocer algunos aspectos de las prácticas de consumo de fauna y de manejo de rebaños por parte de los antiguos pobladores locales. Además de considerar las transformaciones y persistencias a través del tiempo, contemplamos las variaciones funcionales de las ocupaciones analizadas, así como también aspectos contextuales. Los camélidos constituyen la especie más representada en todos los conjuntos, haciendo visible el consumo de recursos pastoriles durante toda la secuencia. Para las ocupaciones de los siglos XIII y XIV hemos registrado camélidos silvestres, siendo muy escasa la presencia de otros taxones en las muestras, mientras que en las ocupaciones fechadas en tiempos coloniales se destaca la ausencia de fauna europea en los contextos excavados. Las clases de edad nos permiten dejar planteada la hipótesis que afirma que se practicaron estrategias mixtas de explotación de recursos, al menos para los siglos XIII y XIV. Finalmente, las semejanzas y divergencias encontradas entre los conjuntos, parecen más bien estar en relación con las particularidades que adquirieron las ocupaciones domésticas.
\end{abstract}

Palabras clave: Zooarqueología; Puna de Jujuy; Pastoralismo; Período Prehispánico; Período Colonial.

\begin{abstract}
The comparative analysis of different zooarchaeological assemblages recovered in sites in the south of the Pozuelos basin (puna de Jujuy, Argentina) dated between the 13th and 18th centuries, allowed us to recognize some aspects of fauna consumption and herd managment practices of the ancient local inhabitants. We will consider the transformations and persistence through time, contemplating the functional variations and contextual aspects of the analyzed occupations. Camelids are the most represented species in all groups, making the consumption of pastoral resources visible throughout the sequence. For the occupations of the thirteenth and fourteenth centuries we have recorded wild camelids, the presence of other taxa in the samples being very scarce, in the colonial occupations the absence of European fauna in the excavated contexts is striking. The age classes suggest that mixed resource exploitation strategies were practiced, at least during the 13th and 14th centuries. Finally, the similarities and differences found between the sets seem to be due to the particularities acquired by the domestic occupations.
\end{abstract}

Key words: Zooarchaeology; Puna de Jujuy; Pastoralism; Pre-Hispanic Period; Colonial Period.

\section{Introducción}

En este trabajo presentamos los resultados del análisis comparativo de nueve conjuntos zooarqueológicos recuperados en sitios del sur de la cuenca de Pozuelos (puna de Jujuy, Argentina), con fechados que los ubican entre los siglos XIII y XVIII de nuestra era. Nos propusimos investigar las prácticas de consumo de fauna de los antiguos pobladores locales, situando las preguntas desde una perspectiva temporal comparativa teniendo en cuenta 
que durante el lapso de estudio el área fue incorporada primero al Tawantinsuyu y posteriormente a la colonia española y considerando la variabilidad funcional de las ocupaciones. El abordaje zooarqueológico de los conjuntos nos permitió avanzar en la discusión en torno a las especies consumidas, la existencia o no de prácticas de caza y pastoreo, las implicancias de la incorporación o no de fauna europea, el manejo de los animales de rebaño en función de los productos deseados, y las pautas de circulación y consumo de recursos animales entre las distintas unidades domésticas y en distintos momentos de sus historias ocupacionales.

En las últimas décadas, la ampliación de los estudios zooarqueológicos para la puna permitió discutir distintos aspectos sobre las economías pastoriles, abordando una gran diversidad de temas. Aquí mencionaremos sintéticamente, aquellos aspectos que se encuentran en relación con nuestros objetivos.

Las investigaciones realizadas en diferentes sitios arqueológicos ubicados en la puna argentina, señalan que los camélidos fueron las principales especies utilizadas a lo largo del tiempo (p.e. Erramouspe et.al. 2017; Mercolli et.al., 2018; Mercolli 2019; Samec et.al., 2014; Olivera, 1997; Yacobaccio et.al. 1997-1998; Yacobaccio, 2010, 2012, 2020; Yacobaccio y Vilá, 2016). Las prácticas de caza y pastoreo variaron en sus características e intensidad al ritmo de los cambios ocurridos hacia el interior de las poblaciones locales y en articulación con coyunturas micro y macroregionales, muy discutidas en la literatura arqueológica (p.e. Albeck 2001; Izeta 2008; Moreno 2010; Nielsen 2004, 2006a, 2006b; Olivera y Grant 2008; Raffino 2007; Yacobaccio 2012).

Al analizar las características de las prácticas de caza y su articulación con el pastoreo, son conocidos los modelos explicativos en términos de proceso en los que se señala una disminución de la caza en reacción al afianzamiento del pastoralismo como estrategia dominante (Olivera 1997; Yacobaccio et.al. 1997-98; Yacobaccio y Madero 2001). En este contexto, cabría esperar que la caza se haya articulado con la conservación de los animales de rebaño en la medida en que permitió obtener recursos animales sin sacrificar llamas, minimizado de esta manera los riesgos relacionados a su manejo (Grant y Escola, 2015; Olivera y Grant, 2009; Yacobaccio et.at. 19971998).

Para momentos de la ocupación inkaica del Noroeste Argentino, se ha propuesto que el imperio incluyó estrategias orientadas a la intensificación de la producción minera, agropastoril y artesanal (Williams y D'Altroy, 1998). Sin embargo, diversos autores (como por ejemplo Williams, 2000) relativizan la idea de un alto control, reconociendo que el gobierno fue flexible, variando sus políticas entre las regiones. En este sentido, la caza de vicuñas podría haber quedado bajo el control del inkario
(Puló, 1998; Moreno, 2010; Ratto, 2003; Yacobaccio et.al. 2007) o, por el contrario, que éste no haya ejercido un control tan estricto sobre las poblaciones locales, periféricas al control imperial (Mercolli 2019), situación que habría posibilitado la continuidad de los modos de vida tradicionales (Yacobaccio, 2009). Para momentos coloniales, diferentes registros documentales hacen referencia a la importancia de la vicuña en relación con el pago del tributo impuesto a las poblaciones indígenas locales (Lema, 2006; Moreno, 2010; Moreno y Lema, 2012; Palomeque, 1995; Yacobaccio 2007).

Al analizar las especies representadas en sitios de regiones cercanas a la nuestra, podemos mencionar los trabajos en el área de Susques, donde diferentes autores mencionan ocupaciones de tipo temporario, con estrategias económicas ligadas al pastoreo y la caza, con conjuntos zooarqueológicos compuestos por camélidos domésticos y silvestres, roedores, cánidos, y con cápridos para momentos coloniales (Yacobaccio et.al. 2011). También para el área de Susques, Erramouspe et.al. (2017), describen los hallazgos de Alero Caído 1, que habría sido un lugar de paso y/o puesto de altura de grupos caravaneros, donde también se encuentran representados camélidos domésticos y en menor proporción vicuñas. En el área de Cochinoca, Mercolli y coautores (2018), trabajaron sobre una muestra proveniente de un poblado residencial con una larga secuencia de ocupación, compuesta en su mayoría por camélidos silvestres y domésticos, quedando integrado el conjunto con proporciones notoriamente bajas de roedores, aves, cánidos, cérvidos y armadillos. Vaquer y colaboradores (2018) señalan que en las ocupaciones más tempranas de Casas Quemadas (Cusi Cusi) hay un claro predominio de camélidos, mientras que en los niveles más recientes se han recuperado camélidos como especie mayoritaria y Bos Taurus, para la ocupación fechada a fines del siglo XVII.

Para caracterizar las economías pastoriles se han planteado modelos centrando las prácticas de manejo de rebaños de llamas de acuerdo a los tipos de recursos/ productos para los cuales son criados los animales (e.g. Madero, 2002; Mengoni Goñalons y Yacobaccio, 2006; Wing 1975; Yacobaccio 2007, 2010). Son clásicos los modelos de matanza selectiva, en donde una estrategia orientada a la adquisición de productos postmortem, seleccionaría preferentemente individuos de edad inferior o igual a aquella de máximo crecimiento corporal, lo que resultaría en un perfil etario dominado por crías y animales jóvenes menores a los 36 meses. Por el contrario, una estrategia tendiente a obtener fibra/carga de la cría del rebaño, buscaría mantener los animales vivos, siendo la expectativa arqueológica un perfil etario con proporciones mayores de animales adultos (Madero, 2002; Wing 1975; Yacobaccio 2007).

Al respecto, las investigaciones etnoarqueológicas de 
Yacobaccio y equipo en la localidad de Susques, puna de Jujuy, indican que los modelos productivos que mejor caracterizan el pastoreo andino son esencialmente mixtos (Yacobaccio 2007; Yacobaccio et.al. 1997-98; Madero, 2002), situación que se caracteriza por el interés en la obtención de productos tanto post-mortem (carne), como ante-morten (fibra), con énfasis variable en uno $u$ otro según factores económicos y ambientales (Yacobaccio, 2007). Göbel (2001) por su parte, menciona que las decisiones de matanza muchas veces varían en escalas de tiempo relativamente cortas en términos arqueológicos, por lo que la posibilidad de enfrentarse a perfiles de edad que agrupen distintas estrategias de matanza es muy alta.

Teniendo en consideración estas propuestas de matanzas selectivas, podríamos utilizarlas como referentes empíricos a partir de las cuales contrastar patrones locales, pero recordando que no deben considerarse "tipos puros". Es decir, que su valor radica en que ofrecen un punto de referencia básico a partir del cual explorar las variaciones observadas en los casos concretos, sin esperar que la cría de animales haya estado orientada hacia la optimización de algún producto específico (Yacobaccio, 2007).

Yacobaccio et. al. (1997-1998) plantean que entre los pastores actuales de Susques, los patrones de descarte muestran un predominio en la representación del esqueleto axial por sobre el apendicular, siendo éste el patrón esperable en contextos de pastoreo dominante. Agregan que en los sitios de ocupación temporaria es esperable encontrar una mayor cantidad de huesos con alto contenido de carne y bajo contenido de médula ósea, mientras que en las bases residenciales, existiría una distribución más proporcional (Yacobaccio et.at. 1997-1998: 345). Si tomamos algunos ejemplos arqueológicos, encontramos similitudes y variaciones en Puesto Demetria, para el cual Yacobaccio et. al. (19971998) afirman que hay un claro predominio del esqueleto axial por sobre el apendicular, mientras que en Cueva Chayal, se da la situación inversa. En Cueva Quispe, Yacobaccio et. al. (2011) y Yacobaccio (2020) señalan que el perfil de unidades anatómicas seleccionadas estaría señalando la incorporación de partes con un rendimiento relativamente balanceado en carne y médula, cocinadas mediante hervido, situación que contrasta con los puestos temporarios actuales. En las ocupaciones fechadas en el tardío-inka de Casas Quemadas (Vaquer et.al. 2018) predomina el esqueleto apendicular, mientras que en la ocupación fechada a fines del siglo XVII, esta diferencia no es tan notoria ni clara. En el poblado de Cochinoca, Mercolli et. al. (2018), analizan la muestra proveniente de un espacio de descarte comunitario, donde todas las partes están representadas, lo que da cuenta del aprovechamiento integral de los camélidos.

\section{El área de estudio y antecedentes de investigación}

La cuenca intermontana de Pozuelos es una depresión de aproximadamente $100 \mathrm{~km}$ de largo por unos de 20 $\mathrm{km}$ de ancho delimitada por las sierras de Rinconada y Carahuasi al oeste y la sierra de Cochinoca-Escaya, al este. Nuestra área de estudio se ubica en el sector sur de la cuenca y se conforma por el fondo de cuenca, formaciones montañosas y terrazas (Figura 1).

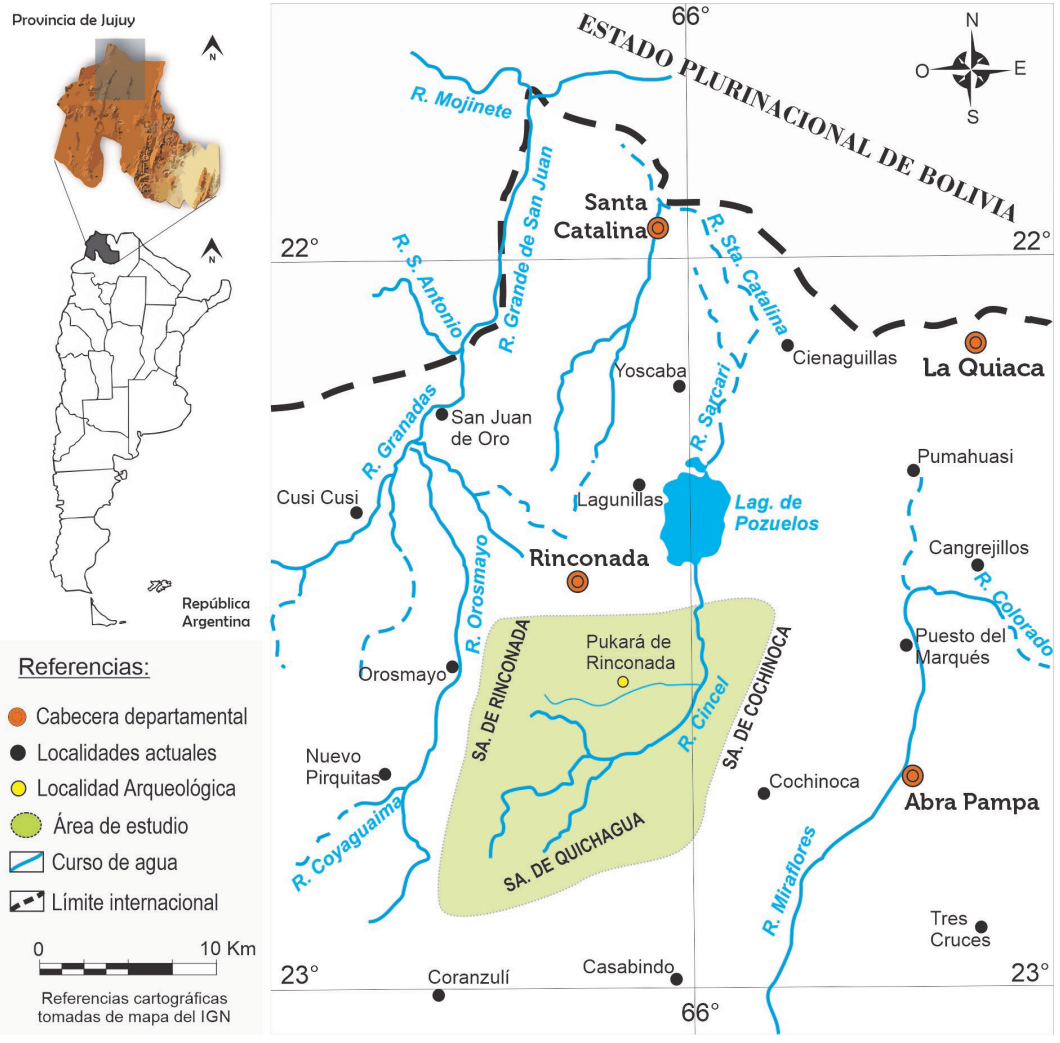

Figura 1. Área de estudio. Mapa confeccionado por S. Rodríguez Curletto.

Figure 1: Study Area. Map made by S. Rodríguez Curletto. 
Desde el año 2004, desarrollamos en el área tareas de investigación que nos permitieron identificar numerosos sitios arqueológicos, proponer un marco cronológico para las evidencias halladas, reconocer los modos de uso del espacio regional y las relaciones entre las ocupaciones de esta zona y áreas vecinas para cada momento (Angiorama 2011), y evaluar el impacto de las conquistas inka y española en el área (Angiorama et.al. 2018a; Angiorama et.al. 2019, entre otros).

Para fines del primer milenio de nuestra era e inicios del segundo, la población local estuvo distribuida por el territorio de manera no uniforme, en pequeños caseríos dispersos, emplazados en escenarios apropiados para el desarrollo de actividades pastoriles y agrícolas, con numerosos lugares de ocupación temporaria distribuidos por la cuenca (Angiorama 2011). El Pukará de Rinconada y Moreta se constituyeron como los poblados más grandes y complejos del área (Angiorama 2011, Angiorama et.al. 2019; Ruiz y Albeck 1997).

La incorporación de Pozuelos al Tawantinsuyu provocó transformaciones importantes en las redes de tráfico interregional, en la arquitectura de sitios como Moreta y el Pukará de Rinconada, y en la organización de la producción agrícola de las tierras aledañas (Angiorama et.al. 2017, Rodríguez Curletto y Angiorama 2019). Sin embargo, las poblaciones dispersas de otros sectores de la cuenca, no habrían sufrido cambios profundos en su modo de vida (Angiorama 2011; Angiorama et.al. 2017).

Las ocupaciones dispersas de tiempos coloniales presentan, en general, características arquitectónicas, de emplazamiento y materiales asociados muy similares a los datados para momentos prehispánicos (Angiorama 2011). Solo los fechados radiocarbónicos y la presencia de unos pocos bienes nos permiten asignarlos a momentos posconquista (Angiorama y Pérez Pieroni 2012). Los trabajos arqueológicos permitieron confirmar la continuidad de la ocupación, en algunos casos, o la reocupación, en otros, de ciertas estructuras habitacionales y asentamientos prehispánicos durante época colonial (Angiorama et.al. 2017; Angiorama et.al. 2018a, Pérez Pieroni y Angiorama, 2018). En este sentido, en ámbitos rurales de nuestra área de estudio ciertos aspectos de los modos de vida de sus habitantes se mantuvieron con pocas transformaciones durante mucho tiempo, a pesar de las conquistas inkaica y española (Angiorama et.al. 2017; Angiorama et.al. 2018a; Pérez Pieroni y Angiorama, 2018).

\section{Procedencia de las muestras arqueofaunísticas estudiadas}

El material faunístico analizado fue recuperando a partir de la excavación de seis recintos domésticos completos y de un sondeo realizado en un basurero, localizados en sectores de fondo de cuenca y quebradas intermedias del sur de Pozuelos. Los contextos excavados fueron fechados mediante radiocarbono entre los siglos XIII y XVII de nuestra era, y son los descriptos a continuación.

La localidad arqueológica de Río Herrana $(\mathrm{RH})$ comprende una serie de terrazas de ignimbrita ubicadas a lo largo del río homónimo, a unos 3.800 msnm. El Pukará de Rinconada se emplaza en la cima de una de estas terrazas, y en el resto del área se distribuyen estructuras agrícolas, corrales, pequeños caseríos, recintos habitacionales aislados, refugios construidos bajo aleros y numerosos paneles con arte rupestre (Angiorama 2011; Rodríguez Curletto y Angiorama, 2019).

Río Herrana 19 (RH19) es uno de los sitios registrados en esta localidad, y está conformado por ocho recintos circulares, canchones agrícolas, muros para la contención del terreno y montículos de despedre (Angiorama et.al. 2018b, 2018c). La Estructura 7, excavada completa, es un recinto habitacional doméstico en el que se registraron dos niveles de ocupación sucesivos. El nivel 2 (N2), más antiguo, fue fechado entre el 1216-1294 cal. AD 1, 2. El nivel 1 (N1), más moderno, fue fechado entre los años 1287-1389 cal. AD. (Angiorama y Pérez Pieroni 2012). En ambos casos, la abundancia, diversidad y características de los hallazgos efectuados sugieren que las ocupaciones habrían conformado bases residenciales.

Río Herrana 10 ( $\mathrm{RH} 10$ ) es un sitio compuesto por andenes y canchones agrícolas, montículos de despedre, refugios construidos bajo bloques, unos pocos recintos habitacionales aislados y un bloque con abundante arte rupestre grabado (Rodríguez Curletto y Angiorama 2019). La excavación de la Estructura 1 nos permitió determinar que fue construida en tiempos prehispánicos tardíos y remodelada durante época colonial y que fue utilizada con fines domésticos, posiblemente como puesto de ocupación temporario a juzgar por los escasos hallazgos realizados. Los fechados radiocarbónicos indican que el primer episodio de ocupación tuvo lugar entre los años 1327-1445 cal. AD (N2), mientras que el segundo ocurrió en el lapso comprendido entre 1649 y 1812 cal. AD (N1) (Angiorama y Pérez Pieroni 2012; Pérez Pieroni 2013).

Tabladitas 1 (Ta1) es un pequeño caserío localizado a $3.800 \mathrm{msnm}$, en el sector medio de una quebrada que comunica la cuenca de Pozuelos con el sur. El sitio está compuesto por unos pocos recintos habitacionales de planta circular, canchones agrícolas, chullpas construidas en oquedades de las peñas (hoy destruidas) y arte rupestre pintado (Rodríguez Curletto y Angiorama 2019). La Estructura 1, excavada completa, es un recinto de planta circular con una única ocupación datada entre el 13121432 cal. AD, en el que se hallaron evidencias materiales

\footnotetext{
1 En todos los casos los fechados radiocarbónicos han sido calibrados a 1 sigma con el programa CALIB 7.0.4; Calibration data set: shcal13.14c (Hogg et al. 2013).

2 Los fechados mencionados fueron originalmente publicados en Angiorama y Pérez Pieroni 2012 y Pérez Pieroni 2013.
} 
de actividades domésticas. Las características de los hallazgos y su abundancia indican que posiblemente se trató de una base residencial.

Chajarahuayco 25 (Ch25) se ubica en una quebrada que comunica la cuenca de Pozuelos con el oeste, a unos $3.600 \mathrm{msnm}$. El sitio se compone de cuatro estructuras rectangulares asociadas a un complejo de andenes agrícolas que ocupan media hectárea, irrigados por al menos un canal, situación excepcional en el sur de Pozuelos (Angiorama et.al. 2018b, 2018c). Las dataciones obtenidas a partir de la excavación de dos recintos completos y las variedades cultivadas en los andenes agrícolas (Angiorama et. al. 2018c) nos permiten señalar que Ch25 fue construido en época inkaica y reocupado durante época colonial. La Estructura 2 (Ch25-E2), excavada completa, es un recinto con un único episodio de ocupación, fechado por radiocarbono entre 14151498 cal. AD (Angiorama y Pérez Pieroni, 2012). La Estructura 1 (Ch25-E1), también excavada en su totalidad y con un solo nivel de ocupación, arrojó un fechado radiocarbónico "moderno", pero es muy factible que haya sido habitada hace más de 200 años. Por un lado, porque presenta las mismas características arquitectónicas, estado de conservación y cobertura de líquenes que la Estructura 2, localizada junto a ella y fechada en el siglo XV. Sin embargo, no proponemos una ocupación prehispánica para esta estructura, principalmente por las características de algunos fragmentos cerámicos hallados en ella, de cronología colonial (Angiorama et. al. 2018a; Pérez Pieroni 2013). Además, reforzando su asignación temporal, junto al sitio se encuentran los restos de un horno de fundición metalúrgica de más de 300 años de antigüedad, y en los niveles superiores de andenes sondeados se han hallado fitolitos de Triticum sp. (trigo), confirmando la reutilización de las estructuras de cultivo durante época colonial (Angiorama et. al. 2018b, 2018c; Angiorama y Pérez Pieroni, 2012). La reducida cantidad y variedad de hallazgos efectuados en los dos recintos excavados, sumado a la inexistencia de basureros en el sitio, indican que se trataría de puestos de ocupación temporaria (Angiorama et. al. 2018c; Angiorama y Pérez Pieroni 2012).

Casa Colorada se encuentra ubicada a 3.500 msnm, en las cercanías del Abra de Queta, el principal paso desde la cuenca de Pozuelos hacia el sur. El sitio Casa Colorada 2 (CCO2) está conformado por cuatro estructuras de planta circular y canchones agrícolas de gran tamaño. La Estructura 1, excavada completa, presenta una única ocupación de carácter doméstico fechada por radiocarbono entre el 1421-1507 cal. AD. Los hallazgos sugieren que se habría tratado de un puesto de ocupación temporaria.

En la localidad arqueológica Pan de Azúcar, donde se encuentra el poblado homónimo actual, se recuperaron evidencias de una larga ocupación. De tiempos prehispánicos tardíos datan algunas estructuras habitacionales aisladas, corrales y estructuras de cultivo, así como también un lugar de prácticas rituales en la cima del cerro. Posteriormente, en tiempos coloniales, Pan de Azúcar adquirió una gran importancia, fundamentalmente por su riqueza en minerales de plata. Se han hallado en el lugar abundantes evidencias de prácticas mineras y metalúrgicas (Angiorama et. al. 2015). Pan de Azúcar 22 (PA 22) es un basurero, parcialmente erosionado por un curso de agua, que se extiende por un área de al menos $14 \mathrm{~m}$ de diámetro. No se encuentra asociado directamente a alguna estructura en particular, pero se emplaza en un sector con recintos y corrales, donde abundan en superficie materiales prehispánicos y, sobre todo, coloniales. Realizamos un sondeo estratigráfico que permitió fechar su nivel más moderno entre 1640 y 1810 cal. AD (Angiorama y Pérez Pieroni 2012).

\section{Metodología}

Para el análisis arqueofaunístico utilizamos una metodología pertinente para responder los interrogantes y objetivos planteados. Las muestras seleccionadas para este trabajo se agrupan en nueve conjuntos, asignables cada uno de ellos a un nivel de ocupación de los recintos excavados, y al basurero en el caso de PA22. Es decir, dos conjuntos de restos arqueofaunísticos provienen de la Estructura 7 de Río Herrana 19 y dos de la Estructura 1 de Río Herrana 10 (que presentan dos pisos de ocupación cada una), mientras que a cada uno de los recintos habitacionales restantes corresponden un solo conjunto, vinculado en cada caso a un único piso de ocupación. Cada conjunto fue definido a partir de la totalidad de los materiales óseos provenientes de cada piso de ocupación arqueológica, y por lo tanto fueron analizados de forma independiente y posteriormente cotejados.

Para evaluar la conservación y el estado de las muestras, consideramos primeramente la meteorización sensu Behrensmeyer (1978), la presencia de marcas de origen natural y antrópico (marcas de corte) y las termoalteraciones (Mengoni Goñalons 2013).

Para obtener resultados respecto a la abundancia taxonómica, utilizamos los índices de NR (Número Total de Restos Óseos), NISP (Número de Especímenes Identificados por Taxón), y un NISP discriminado por parte esqueletaria. Los especímenes óseos que no pudieron asignarse a una categoría taxonómica se consideraron como no identificables (NID) (Grayson 1984).

Para identificar y discutir la presencia de especies al interior del grupo Camelidae, utilizamos las técnicas de mayor difusión basadas en análisis morfológico comparativo, principalmente de dientes incisivos (Wheeler 1982), y cuando fue posible, utilizamos variables osteométricas sobre elementos óseos arqueológicos asignados anatómicamente a falanges proximales que, 
además, muestran madurez ósea y no presentan signos de termoalteración, meteorización o patologías. Para cada falange se tomaron dos medidas (ancho máximo y espesor de la cara articular de la epífisis proximal) (Kent 1982). En este caso,

\begin{tabular}{|c|c|c|c|c|c|c|c|c|c|c|}
\hline & $\begin{array}{c}\mathrm{RH} 19 \mathrm{~N} \\
2\end{array}$ & $\begin{array}{c}\mathrm{RH} 19 \\
\mathrm{~N} 19\end{array}$ & Ta1 & $\begin{array}{c}\text { Rh10 } \\
\text { N2 }\end{array}$ & $\mathrm{CCO} 2$ & $\begin{array}{c}\text { Ch25 } \\
\text { E2 }\end{array}$ & $\begin{array}{c}\text { Ch25 } \\
\text { E1 }\end{array}$ & PA22 & $\begin{array}{c}\text { Rh10 } \\
\text { N1 }\end{array}$ & Total \\
\hline A & - & 77 & 36 & 2 & 16 & 2 & - & 45 & 13 & 191 \\
\hline Cam & 155 & 56 & 57 & 15 & 9 & 7 & 5 & 1 & 5 & 310 \\
\hline$A v$ & - & 2 & 1 & - & - & - & - & - & - & 3 \\
\hline Ro & 1 & 1 & - & - & - & - & - & - & - & 2 \\
\hline $\begin{array}{l}\text { Subtotal } \\
\text { NISP }\end{array}$ & 156 & 136 & 94 & 17 & 25 & 9 & 5 & 46 & 18 & 506 \\
\hline NID & 295 & 252 & 85 & 31 & 43 & 2 & & 56 & 80 & 844 \\
\hline Total & 451 & 388 & 179 & 48 & 68 & 11 & 5 & 102 & 98 & 1350 \\
\hline
\end{tabular}
utilizamos como referencia las medidas tomadas sobre falanges de dos vicuñas de Abra Pampa (Mengoni Goñalons y Elkin com. pers.; Mercolli 2009a), un guanaco de los Valles Calchaquíes (Mengoni Goñalons y Elkin com. pers.) y una llama de Rinconada (Mercolli 2009b).

Para comenzar a discutir aspectos relacionados con el consumo de partes esqueletarias, utilizamos un NISP por parte esqueletaria y el esquema propuesto por Mengoni Goñalons (2013) para evaluar la utilidad económica de las diferentes partes anatómicas.

Para la determinación de perfiles etarios de camélidos utilizamos dos criterios complementarios: las secuencias de brote, reemplazo y desgaste en series mandibulares y maxilares (Wheeler 1982; Puig 1988; Puig y Monge 1983), y el estado de fusión de los centros de osificación (utilizamos un esquema propuesto por Mengoni Goñalons 2013, basado en Kent 1982), lo que nos permitió asignar a los restos una categoría etaria y aproximarnos a la edad de muerte de los animales. En este sentido, la información que las secuencias de erupción y remplazo dentaria ofrece mayor resolución, ya que permite establecer un rango de edad de muerte definido para cada espécimen analizado, mientras que las evidencias de fusión ósea, permiten establecer edades máximas de muerte solo hasta la madurez osteológica (Mengoni Goñalons 1999), por lo que posee una menor resolución y se restringe a los primeros tres o cuatro años.

La secuencia de brote y remplazo de dientes nos permitió dividir la muestra en cuatro categorías de edad (con sus respectivas subcategorías): Crías (0 a 12 meses), Juveniles (12 a 24 meses), Subadultos (24 a 36 meses) y Adultos (36 a 168 meses) (Puig y Monge, 1983). Complementariamente, utilizamos un esquema propuesto por Mengoni Goñalons (2013) para la fusión de huesos largos, que se resuelve en tres etapas: (a) fusión temprana ( $<12$ a 18 meses), (b) fusión intermedia $(<18$ a 36 meses) y (c) fusión tardía (<36 a 48 meses), según la edad mínima en que ocurre la unión de los diferentes centros de osificación. Consecuentemente, se definen dos grupos de huesos, no fusionados y fusionados, que se asocian a animales inmaduros y animales maduros $\mathrm{o}$ adultos respectivamete. Este procedimiento, permite definir distintas clases de edad y considerar las frecuencias de elementos fusionados y no fusionados de cada una de esas edades (Yacobaccio, 2007, Mengoñi Goñalons, 2010), interpretadas como medidas de mortalidad y supervivencia para cada intervalo temporal.

Tabla 1. Composición taxonómica de la muestra estudiada. Abreviaturas: A: Artiodáctilos indeterminados; Cam: Camélidos; Av: Aves; Ro: Roedores.

Table 1. Taxonomic composition of the studied sample. Abbreviations: A: Indeterminate Artiodactyls; Cam: Camelids; Av: Birds; Ro: Rodents.

\section{Resultados}

Sobre un total de 1350 especímenes óseos analizados, 506 elementos (37\%) han podido ser identificados, al menos a nivel de Orden/Familia. Los resultados de las observaciones sobre meteorización, marcas y termoalteraciones nos muestran la prevalencia de los estadios de meteorización "0" y "1" (68,1\%), la bajísima frecuencia de marcas de origen natural $(\mathrm{N}=1)$ y de procesamiento, registrando marcas de corte solo en cinco elementos, y por el predominio de especímenes no termoalterados (85,05\%). Sin embargo, reconocemos una baja identificabilidad en los conjuntos, por lo que no debemos descartar que otros factores o procesos pudieron haber afectado el conjunto, considerando la alta frecuencia de astillas.

Los restos óseos de camélidos y artiodáctilos indeterminados dominan los conjuntos, superando el $98 \%$ del total de la muestra. Dado que los camélidos son los únicos artiodáctilos identificados, es posible que los especímenes integrados en la categoría artiodáctilo indeterminado, pertenezcan a camélidos, por lo que la discusión estará centrada en el rol de los mismos.

El conjunto fechado en el siglo XIII, corresponde a la primera ocupación de RH19-N2 se compone de 451 especímenes óseos, predominando los de Camélidos con un $99,4 \%$ (Tabla 1). Se analizaron cuatro incisivos sueltos que de acuerdo a los caracteres morfológicos poseen rasgos asociados a llama/guanaco. La estimación de la edad de los mismos los ubica en un rango de los cero a siete días ("Crías 1") y entre los 12 a 18 meses ("Juvenil $1 "$ ). Este resultado es coherente con lo que indica el análisis de los perfiles etarios, ya que la mayoría de los especímenes se agrupan en la categoría no fusionados. En los perfiles se observa la presencia de especímenes comprendidos en las categorías de edad de crías, juveniles y subadultos, lo cual implicaría que el sacrificio se efectuó principalmente en animales de no más de 36 meses de edad (Figura 2). 


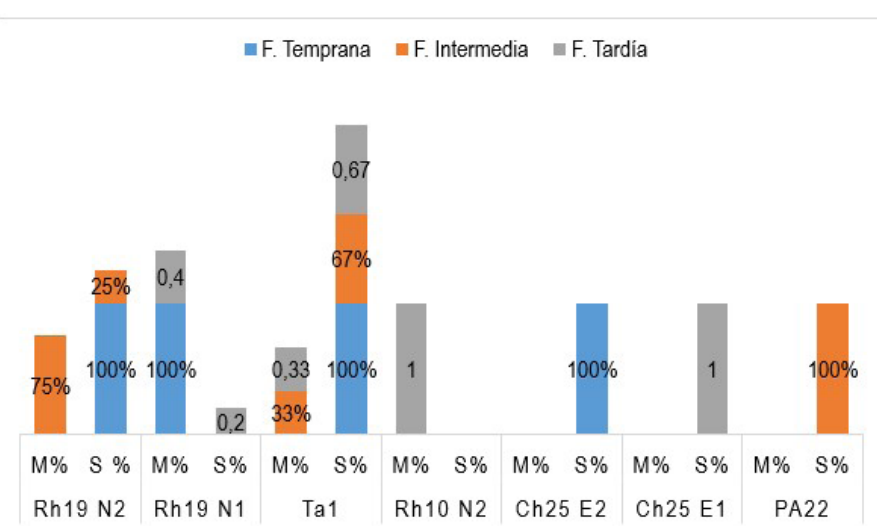

Figura 2. Mortandad-supervivencia de camélidos expresada en porcentajes. Abreviaturas: F.: fusión; M\%: mortandad; S\%: supervivencia.

Figure 2. Mortality-survival of camelids expressed in percentages. Abbreviations: F: fusion; M\%: mortality; S\%: survival.

Las partes esqueletarias señalan un predominio de los elementos asignables al esqueleto apendicular por sobre el axial. Si tenemos en cuenta las partes anatómicas y su utilidad, hay cierta predominancia hay cierta predominancia de las partes/secciones distales de las patas y del cráneo, las cuales proveen médula y seso. Le siguen los huesos con solo carne (vértebras, costillas, pelvis y escápula) y los de las partes medias de las patas y la mandíbula, que proveen poca carne y médula. Los menos abundantes son los huesos correspondientes a las partes proximales/altas de las extremidades, con carne y médula en abundancia. Más allá de la predominancia relativa de una sección o de otra, podemos afirmar que se encuentran representadas todas las partes esqueletarias, lo que sugiere el acceso/consumo a toda la gama de rendimiento (carne, grasa, médula, seso). Esta diversidad de partes anatómicas y su relación con la utilidad es concordante con una ocupación de tipo doméstico, en donde las unidades anatómicas podrían haber entrado tanto como carne fresca como seca (Tabla 2).
Con fechados en los siglos XIV y XV, el conjunto de RH19-N1 es el de mayor tamaño ( $N R=388)$, donde el $35 \%$ de la muestra ha podido ser identificada, prevaleciendo los artiodáctilos indeterminados $(56,6 \%)$ y los camélidos $(41,2 \%)$. Este conjunto ha presentado la mayor diversidad taxonómica, ya que identificamos aves y roedores, pero en una proporción notoriamente baja. En los conjuntos de Ta1 (NR=179) y de RH10 (NR=48) prevalecen los camélidos (Tabla 1).

La osteometría de las falanges recuperadas en Ta1 (Figura 3) indica que tres elementos se ubican cercanos a la medida de referencia de una llama actual, mientras que otro lo hace a la de las vicuñas. Por otra parte, el análisis de dientes incisivos sustenta la presencia de vicuñas.

Para el caso de RH19-N1, el análisis de mandíbulas da cuenta de la presencia de animales de entre $10 \mathrm{y}$ 10,5 años, y crías que van de cero a siete días. El único elemento fusionado señala la presencia de un animal que sobrevivió a los 36 y 48 meses, mientras que la mayoría fueron sacrificados antes de los 36 y 48 meses y de los 12 y 18 meses (NISP NF=4). El análisis de la dentición de Ta1 demuestra que se encuentran animales contemplados en las categorías juveniles de 12 a 18 meses y de 18 a 24 meses, mientras que los adultos son de 72 a 108 meses. La representación de especímenes no fusionados y fusionados es concordante con los análisis de series mandibulares, predominando los fusionados sobre los no fusionados, ya que encontramos animales que sobrevivieron más allá de los 12 y 18 meses (NISP=1), entre los 18 y 36 meses (NISP=6), y de los 36 y 48 meses $(\mathrm{NISP}=2)$. También registramos, pero en proporciones menores, elementos que sugieren que los animales murieron antes de los 18 y 36 meses (NISP=3) y de los 36 y 48 meses (NISP=1). Para la ocupación tardía de RH10-N2, considerando el tamaño y las características de la muestra, la fusión de los huesos solo pudo determinarse a partir de una reducida cantidad de elementos, que indican que

\begin{tabular}{|c|c|c|c|c|c|c|c|c|c|c|c|c|c|c|c|c|c|c|}
\hline \multirow[b]{2}{*}{ Utilidad } & \multicolumn{2}{|c|}{ Rh19 N2 } & \multicolumn{2}{|l|}{ Rh19 N1 } & \multicolumn{2}{|l|}{ Ta1 } & \multicolumn{2}{|c|}{ Rh10-2 } & \multicolumn{2}{|l|}{$\mathrm{CCO} 2$} & \multicolumn{2}{|l|}{ Ch25.2 } & \multicolumn{2}{|l|}{ Ch25.1 } & \multicolumn{2}{|l|}{ PA22 } & \multicolumn{2}{|l|}{ Rh10-1 } \\
\hline & NISP & $\%$ & NISP & $\%$ & NISP & $\%$ & NISP & $\%$ & NISP & $\%$ & NISP & $\%$ & NISP & $\%$ & NISP & $\%$ & NISP & $\%$ \\
\hline 1 & 12 & 17.4 & 6 & 20.7 & 10 & 25 & 2 & 18,2 & - & - & 2 & 40 & - & - & - & - & & 240 \\
\hline 2 & 4 & 5.8 & 4 & 13.8 & 5 & 12.5 & 6 & 54,5 & - & - & 2 & 40 & 5 & 100 & 2 & 100 & & 240 \\
\hline 3 & 8 & 11.6 & 7 & 24.1 & 7 & 17.5 & 3 & 27,3 & - & - & 1 & 20 & - & - & - & - & & - \\
\hline 4 & 22 & 31.9 & 7 & 24.1 & 16 & 40 & - & - & 2 & 66.7 & - & - & - & - & - & - & & 120 \\
\hline 5 & 23 & 33.3 & 5 & 17.2 & 2 & 5 & - & - & 1 & 33.3 & - & - & - & - & - & - & & -- \\
\hline Total & 69 & 100 & 29 & 100 & 40 & 100 & 11 & 100 & 3 & 100 & 5 & 100 & 5 & 100 & 2 & 100 & & \begin{tabular}{|l|l}
5 & 100 \\
\end{tabular} \\
\hline
\end{tabular}

Tabla 2. Partes esqueletarias de camélidos e índices de utilidad, expresados en NISP y NISP\% (Tomado de Mengoni Goñalons, 2013). Referencias: 1- Huesos con solo carne (vértebras, costillas, pelvis y escápula); 2- Huesos con mucha carne y médula (húmero y fémur); 3- huesos con poca carne y médula (radioulna, tibia, mandíbula); 4- Huesos con solo médula (autopodio) y 5: Huesos con seso (cráneo).

Table 2. Skeletal parts of camelids and utility indices, expressed in NISP and NISP\% (Taken from Mengoni Goñalons, 2013). References: 1-Bones with only meat (vertebrae, ribs, pelvis and scapula); 2-Bones with a lot of meat and marrow (humerus and femur); 3- bones with little meat and marrow (radioulna, tibia, jaw); 4- Bones with only marrow (autopodium) and 5- Bones with brains (skull). 


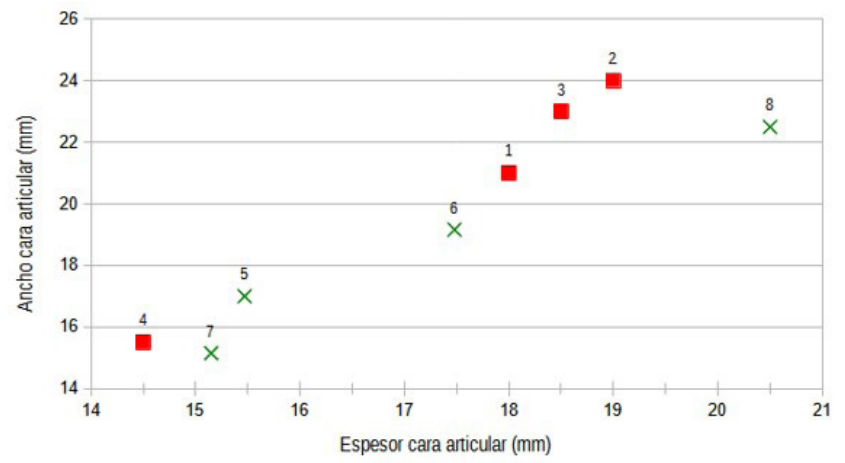

Figura 3. Osteometría de falanges provenientes de Ta1. Referencias: los cuadros rojos, numerados del 1 al 4 , corresponden a las mediciones de las falanges recuperadas en el sur de Pozuelos y las cruces verdes a los estándares de referencia, correspondiendo 5 y 7 a vicuñas (Mengoni Goñalons y Elkin, com.per. y Mercolli 2009b, respectivamente), 6 a guanaco (Mengoni Goñalons y Elkin, com. per.) y 8 a llama (Mercolli, 2009a).

Figure 3. Osteometry of phalanges from Ta1. References: the red squares, numbered from 1 to 4 , correspond to the measurements of the phalanges recovered in the south of Pozuelos and the green crosses to the reference standards, 5 and 7 corresponding to vicuñas (Mengoni Goñalons and Elkin, com.per . and Mercolli 2009b, respectively), 6 to guanaco (Mengoni Goñalons and Elkin, per. comm.) and 8 to llama (Mercolli, 2009a).

los animales no sobrevivieron más allá de los 36 y 48 meses (Figura 2).

En cuanto a la representación de partes esqueletarias, en $\mathrm{RH} 19-\mathrm{N} 1$ (NISP=56) los elementos del esqueleto apendicular $(\mathrm{NISP}=16)$ señalan la presencia de extremidades posteriores en sus tres segmentos, extremidades anteriores en sus partes proximal y media y elementos correspondientes al autopodio. El esqueleto axial (NISP=39), está representado por huesos de la columna, costillas y pelvis. Si bien la relación axial/apendicular estima un predominio del primero, consideramos que la categoría más abundante (dientes sueltos) puede corresponder a pocos individuos y estar sobreestimando la abundancia relativa de uno sobre otro. Si tenemos en cuenta las partes anatómicas y su utilidad, notamos que los elementos que proveen de médula ósea y aquellos que tienen poca carne y médula son los más abundantes en RH19-N1. Sin embargo, es importante señalar que, teniendo en cuenta que se encuentran unidades anatómicas con distintas utilidades, es factible pensar que los antiguos pobladores de RH19 utilizaron todas las partes esqueletarias disponibles (para la obtención de carne, médula y seso) (Tabla 2).

En Ta1 (NISP=57) hemos registrado que el $74 \%$ de la muestra corresponde a restos pertenecientes al esqueleto apendicular (NISP=38), mientras que el 19\% restante corresponde al esqueleto axial (NISP=19).
Entre las unidades anatómicas mayores del esqueleto apendicular, predominan las extremidades traseras (en sus tres secciones) y delanteras (en sus sección media). Del esqueleto axial, las unidades anatómicas representadas son cráneo, pelvis, costillas y vértebras. En Ta1 se percibe una abundancia de aquellas partes que ofrecen solo médula, seguidas por las que proveen mayormente carne. Como en los casos anteriores, también encontramos representadas en bajas proporciones unidades anatómicas de las que pueden extraerse poca carne y médula, así como aquellas de las que se obtiene mucha carne y médula. Finalmente, los huesos del cráneo parecen señalar el consumo de seso. Nuevamente, nos encontramos frente a un escenario en el que el aprovechamiento de médula-carne-seso y demás productos primarios se presenta coherente con la diversidad de unidades anatómicas a las que tuvieron acceso los habitantes de este recinto (Tabla 2).

Entre los restos de camélido de RH10-N2 (NISP=14), encontramos elementos correspondientes a la parte alta y media de las patas traseras (esqueleto apendicular $\mathrm{NISP}=9)$, mientras que la zona axial $(\mathrm{NISP}=5)$ del esqueleto está representada por vértebras indeterminadas y dientes sueltos. El conjunto de RH10, por otra parte, da cuenta de una diferencia en comparación con los otros dos conjuntos fechados para los siglos XIV y XV: en este caso solo tenemos representados elementos correspondientes a unidades anatómicas que proveen mucha carne y médula (fémur), poca carne y médula (tibia) y, finalmente, solo carne (vértebras) (Tabla 2).

Los dos conjuntos zooarqueológicos correspondientes al siglo XV (Ch25-E2 y CCo2) provienen de ocupaciones de tipo doméstico en puestos temporarios y se caracterizan por ser poco numerosos, no obstante lo cual, fue posible identificar restos de camélidos (Tabla 1). Por las características de la muestra de $\mathrm{CCO} 2$ no fue posible avanzar en la construcción de perfiles etarios en base a los criterios de estadios de fusión y de series dentarias, mientras que para Ch25-E2, solo pudimos realizar observaciones sobre el estadio de fusión alcanzado sobre un fragmento de húmero distal que se encuentra fusionado, lo que indica que el animal sobrevivió más allá de los 12 y 18 meses (Figura 2).

En relación a las partes esqueletarias de camélidos identificados en CCO2, hemos podido comprobar la presencia de elementos asignables al basipodio (sección anatómica que provee fundamentalmente médula) y del cráneo, rico en seso y grasa. En el caso de Ch25-E2, entre los huesos del esqueleto axial aparecen representadas solamente vértebras fragmentadas, mientras que el conjunto óseo de la región apendicular está conformado por elementos correspondientes a las extremidades delanteras y traseras. Como vemos, en este caso, la muestra nos señala la utilización de partes esqueletarias que proveen carne y médula, en distintas proporciones (Tabla 2). 
En cuanto a los taxones presentes en los contextos coloniales de los siglos XVII y XVIII, hemos registrado el consumo de camélidos pero no identificamos la presencia de fauna de origen europeo. En el piso de ocupación de Ch25-E1 pudimos reconocer solo cinco elementos, todos correspondientes a un mismo fragmento de tibia proximal de camélido. El conjunto correspondiente a la ocupación colonial de RH10-N1 está conformado por 98 especímenes óseos donde el $18 \%$ ha podido ser identificado, dentro de los cuales el $28 \%(\mathrm{NISP}=5)$ ha sido adscripto a la categoría camélidos, sin haberse podido obtener mayores precisiones sobre morfotipos. En el sondeo del basurero de PA22, finalmente, recuperamos 102 especímenes óseos de los cuales el 45\% (NISP=46) ha podido ser identificado. A excepción de un fragmento de tibia distal de camélido, los restantes elementos se han agrupado en la categoría Artiodáctilo indeterminado (Tabla 1).

Como mencionamos, en Ch25-E1 los elementos recuperados corresponden a una tibia proximal fusionada de camélido, lo que señala que el animal sobrevivió a los cuatro años de edad. En el caso de RH10-N1, no hemos podido obtener mayores precisiones sobre perfiles etarios, al no contar con elementos diagnósticos (Figura 2).

Las unidades anatómicas representadas en los casos de Ch25-E1 y PA22 se limitan a huesos del sector medio de las patas traseras que, a su vez, ofrecen poca carne y médula. Por su parte, para RH10-N1 el esqueleto apendicular está representado por huesos de las extremidades posteriores y al esqueleto axial corresponde una vértebra y la pelvis. A pesar de lo escueto del conjunto, nos muestra cierta diversidad y podemos considerar la posibilidad de que los antiguos reocupantes coloniales de RH10-N1 accedieron a unidades esqueletarias con diferentes utilidades, a diferencia de lo que de algún modo muestran los otros dos conjuntos, en los que solo se encuentran representadas partes anatómicas con poca carne y médula (Tabla 2).

\section{Discusión}

Lo primero que podemos señalar luego del análisis de la muestra de todos los conjuntos es que las ocupaciones domésticas de RH19 y Ta1, datadas en los siglos XIII y XIV, poseen la mayor cantidad de especímenes óseos y diversidad taxonómica. Esto último puede deberse a que el tamaño de la muestra es mayor, lo que a su vez refuerza la idea de que las ocupaciones de RH19 y Ta1 habrían funcionado como bases residenciales. Complementariamente, los conjuntos de RH10-N2, Ch25-E2 y CCO2, datadas en los siglos XIII y XIV, y los de RH10-N1 y Ch25-E1, de tiempos coloniales, con cantidades reducidas de especímenes óseos, estarían apoyando la idea de la función propuesta para estos recintos como puestos temporarios y con baja densidad de ocupación. Cabe recordar aquí que la muestra de PA22 proviene del sondeo de un basurero, lo que la distingue del resto de los conjuntos. Si bien la cantidad de especímenes recuperados allí es proporcionalmente mayor, las especies y taxones representados no muestran divergencias. En este sentido, las variaciones en los tamaños de los conjuntos estarían en relación con las distintas configuraciones de los espacios domésticos.

El segundo de los puntos para destacar es que los camélidos son la especie más representada en todos los conjuntos analizados, lo cual es coincidente con lo descrito para otros sitios de la región con fechados comparables (Carreras 2020; Mercolli et.al. 2018; Vaquer et.al. 2018; Yacobaccio 2010, 2012, 2020, solo por mencionar algunos ejemplos). La baja representatividad de otras especies nos lleva a contemplar las posibilidades de que el interés por las mismas haya sido escaso o casi nulo, y su consumo haya sido netamente circunstancial, o bien, que el grado de fragmentación de la muestra haya impedido su identificación.

En tercer lugar, en los contextos de los siglos XIII y XIV, la identificación de camélidos silvestres estaría dando cuenta de un escenario de pastoreo con prácticas de caza como estrategia económica complementaria. A su vez, la circulación de estos recursos de caza (especialmente vicuña) para este lapso temporal, parece ser homogénea en las bases residenciales, ya que han sido registradas tanto en Ta1 como en RH19-N2. Sin embargo, no fue posible identificar restos asignables a animales silvestres en las ocupaciones de RH10-N2, Ch25-E2 y CCO2. Si en el futuro esta situación se reitera para este tipo de ocupaciones en puestos temporarios, se configuraría como una diferencia en relación a otros sitios señalados como puestos, donde se ha registrado el consumo ocasional de recursos de caza (Erramouspe et.al. 2017; Yacobaccio et.al. 2013). Podría plantearse que las actividades en bases residenciales y puestos temporarios del sur de Pozuelos involucraron prácticas diferentes en este sentido. Sin embargo, la ausencia de camélidos silvestres y de otros recursos de caza en los conjuntos óseos en algunas localidades, no implica necesariamente que las prácticas de caza fueran marginales o inexistentes en la economía de las comunidades que analizamos. En este sentido, parece que el pastoreo ha sido una actividad ubicua mientras que los restos asignados a recursos silvestres (en estos casos vicuñas y aves), fueron registrados solamente en los conjuntos óseos más grandes. Esto indica que la información disponible es insuficiente para definir a la caza como una práctica ocasional o secundaria.

Para momentos coloniales hemos descrito elementos óseos correspondientes a camélidos, sin poder avanzar en la diferenciación interespecífica, por lo que tampoco podemos abordar aquí la continuidad (o no) de prácticas de caza y/o el papel de ésta en la colonia como fuente ingreso de dinero para el pago del tributo, tal como lo proponen Moreno y Lema (2012) para sectores de la 
puna Catamarqueña. Si bien la muestra analizada es pequeña, lo expuesto aquí nos sugiere que hasta los siglos XVII-XVIII, los pastores que habitaron los recintos estudiados no consumían animales europeos de forma regular, aun teniendo acceso a objetos coloniales (como ciertas piezas cerámicas), lo que nos lleva a pensar que el consumo de animales locales quizás fue una elección y que la incorporación de los animales europeos a la dieta se habría consolidado ya en tiempos republicanos (Angiorama et. al. 2019; Vaquer et. al. 2018).

En cuarto lugar, para el siglo XIII los perfiles etarios estarían señalando que el consumo se habría concentrado en crías e individuos juveniles, y en menor medida en adultos. Atendiendo a la cantidad de elementos óseos y dientes sobre los que hemos podido realizar observaciones relativas a las edades, es necesario que guardemos cautela en cuanto a esta interpretación, motivo por el cual no podemos afirmar una tendencia en la orientación económica de los pastores de este segmento temporal, aún cuando la muestra analizada confirme la matanza de animales subadultos, situación que suele asociarse con economías pastoriles orientadas principalmente a la obtención de recursos primarios (Mengoni Goñalons y Yacobaccio, 2006; Yacobaccio 2007, 2010).

Para momentos posteriores (siglo XIV) parece más clara la posibilidad de pensar en estrategias mixtas entre recursos post/ante mortem. En RH19-N1 los resultados sugieren la presencia de animales jóvenes y crías y en menor medida adultos, situación diferente a la de Ta1 y RH10-N2. En Ta1 no tenemos registrada la presencia de crías y sí de individuos juveniles y adultos, mientras que en RH10-N2 solo hemos podido confirmar el consumo de subadultos. Si este esquema se repite en otros sitios arqueológicos de la zona, para el mismo segmento temporal y funcionalidad de las estructuras, sería factible pensar que en las bases residenciales se consumían tanto animales que no han alcanzado la madurez osteológica como animales que sí lo han hecho, mientras que en los puestos y estancias el consumo habría estado orientado a animales subadultos, quizás evitando el sacrificio de llamas destinadas a la producción de fibra y/o el caravaneo. Si esto fuera así, siguiendo lo propuesto por Yacobaccio (2007), es factible pensar que el rebaño haya estado segmentado de acuerdo a los productos que se querían obtener; lo que no implicó necesariamente una "especialización", sino la adopción de estrategias de conservación de animales en el marco de modalidades productivas mixtas de carne y fibra. Del mismo modo, la cacería de fauna silvestre podría enmarcarse dentro del conjunto de estrategias desplegadas con el fin de mitigar el sacrificio del rebaño y acceder a recursos primarios, situación que parece haber sido circunstancial. Finalmente, para el siglo XV en adelante, hemos podido probar la presencia de camélidos adultos y subadultos en las muestras, pero no podemos arriesgar apreciaciones acerca de la posibilidad de la existencia de rebaños especializados en la producción de fibra u otros recursos secundarios.

En las ocupaciones de las bases residenciales fechadas en el siglo XIV (RH19-N1 y Ta1), encontramos un escenario similar, ya que si bien predominan los restos del esqueleto apendicular de camélidos, aparecen representadas todas las unidades anatómicas mayores, lo que nos lleva a pensar que sus habitantes accedieron y utilizaron todos los recursos primarios a partir del aprovechamiento integral de las carcasas. Esta diversidad de partes es concordante con ocupaciones de tipo doméstico y es la esperable para bases residenciales con una alta estabilidad ocupacional.

Por su parte, en las muestras provenientes de los sitios de ocupación temporaria, como $\mathrm{RH} 10$, solo recuperamos elementos correspondientes a unidades anatómicas que proveen mucha carne y médula, poca carne y médula y solo carne. Esta situación se repite (y en proporciones similares) en Ch25-E2 para el siglo XV. Lo propio ocurre en CCo2, contemporáneo, en donde registramos cierta divergencia en relación a los perfiles anatómicos, ya que solo hemos podido reconocer elementos correspondientes a unidades anatómicas cuyas utilidades se traducen en seso y médula.

Por otra parte, resulta difícil evaluar las consecuencias que ocasionó la incorporación de estas comunidades al imperio inkaico a partir del registro zooarqueológico analizado. La anexión de Pozuelos al Tawantinsuyu parece no haber modificado la situación previa, ya que lo conjuntos óseos, o bien mantienen semejanzas en cuanto a partes representadas, o las divergencias no parecen estar en relación con la dominación imperial, sino más bien, con las distintas particularidades que adquirieron las ocupaciones domésticas en estos espacios caracterizados como puestos temporarios, situación que se fortalece por lo mencionado por Angiorama et.al. (2017).

En los conjuntos con fechados postconquista española también predominan los restos del esqueleto apendicular, aunque a diferencia de los contextos prehispánicos, solo podemos dar cuenta del aprovechamiento de ciertas unidades anatómicas que proveen poca médula y poca carne para las tres ocupaciones, mientras que el conjunto de RH10-N1 agrega la presencia de otras unidades con mucha carne y médula, solo carne y solo médula. Esta mayor diversidad en las partes representadas y sus utilidades puede estar en relación con distintos aspectos, entre ellos, que se trata de uno de los conjuntos más numerosos, pero también, con la historia ocupacional de este recinto, donde se llevaron a cabo una multiplicidad de actividades domésticas.

En todos los casos, las unidades anatómicas representadas sugieren que las partes pueden haber ingresado como carne fresca y como carne seca, considerando lo planteado por Yacobaccio (2020), quien señala que 
entre los pastores actuales de la zona de Susques, la carne charqueada es lo remanente de lo consumido, distribuido o vendido.

\section{Consideraciones finales}

La información generada a partir del estudio de los conjuntos arqueofaunísticos presentados nos permitió establecer algunas aproximaciones en cuanto a la explotación de la fauna en el sur de la cuenca de Pozuelos. En este sentido, los camélidos fueron el recurso faunístico utilizado por excelencia y la presencia de otros animales resulta sumamente escasa y se limita a los contextos fechados en el siglo XIII. Las diferencias registradas en cuanto a la utilización de camélidos silvestres en función de distintos contextos domésticos abre la posibilidad de considerar que la distribución o consumo de estos productos hayan estado afectados por las unidades sociales implicadas (Mengoni Goñalons 2013). Como agenda a futuro, consideramos necesario llevar a cabo estudios más detallados a nivel intrasitio, así como también incorporar otras líneas de evidencia, para avanzar en este problema.

Las clases de edad que reflejan los conjuntos nos permiten dejar planteada la hipótesis que afirma que se practicaron estrategias mixtas de explotación de recursos a partir del pastoreo, al menos para los siglos XIII y XIV.

Si bien la llegada de los españoles a la región implicó importantes transformaciones para las poblaciones locales, sabemos que no tuvo consecuencias uniformes para todos. La continuidad y la recurrencia de la ocupación de puestos y estancias rurales, con bienes y recursos similares a los registrados para momentos previos a la llegada de los conquistadores, sugiere que existió una perduración de ciertas prácticas de origen prehispánico, al tiempo que las poblaciones locales se integraban e interactuaban en el mundo colonial. De esta manera, el pastoralismo se constituyó como una de las actividades principales de los habitantes del área (Gil Montero 2004, 2015; Angiorama et.al. 2018a).

Los estudios arqueofaunísticos que llevamos a cabo permitieron definir de forma preliminar algunas características de las prácticas de pastoreo y caza desde la perspectiva ofrecida por los materiales óseos asociados a las ocupaciones domésticas dispersas, y conforman así un aporte a la comprensión de este aspecto de la historia local, al mismo tiempo que contribuyen al incremento del conjunto de información zooarqueológica generada para esta parte de la puna. Somos conscientes de que algunos de los problemas abordados precisan una mayor base empírica y la incorporación de otras líneas de evidencia. Trabajos en curso permitirán en el futuro avanzar en las problemáticas señaladas en este artículo.

San Miguel de Tucumán, Yerba Buena y Tilcara, 03 de Agosto de 2021

\section{Agradecimientos}

Agradecemos especialmente a los habitantes de la cuenca de Pozuelos, por su permiso y colaboración para que nuestros estudios hayan podido llevarse a cabo durante casi dos décadas. Damos las gracias también a cada uno de los miembros del equipo que han participado en los trabajos de campo y de laboratorio. Las investigaciones han sido financiadas por subsidios PIP CONICET, PICT FONCYT y PIUNT.

\section{Bibliografía citada}

Albeck, M.E. (2001). La Puna argentina en los períodos medio y tardío. En: Historia Argentina Prehispánica. E. Berberián y A. Nielsen (Eds.): pp. 347-388. Editorial Brujas.

Angiorama, C.I. (2011). La ocupación del espacio en el sur de Pozuelos (Jujuy, Argentina) durante tiempos prehispánicos y coloniales. Estudios Sociales del NOA 11:125-142.

Angiorama, C.I. y M.J. Pérez Pieroni. (2012). Primeros estudios sobre manufacturas cerámicas de contextos coloniales del sur de Pozuelos (Puna de Jujuy). Revista de Arqueología Histórica Argentina y Latinoamericana 6: 95-126.

Angiorama, C.I.; M.F. Becerra y M.J. Pérez Pieroni. (2015). El mineral de Pan de Azúcar. Arqueología histórica de un centro minero colonial en la puna de Jujuy (Argentina). Chungara 47(4):603-619. Arica.

Angiorama, C.I., M.J. Pérez Pieroni y M.F. Becerra. (2017). Moreta, "pueblo de yndios chichas y tambo del ynga" (Puna de Jujuy, Argentina). Estudios. Atacameños 55: 163-181.

Angiorama, C.I, M.J Pérez Pieroni, M.F. Becerra y M. Giusta. (2018a). Cambios y continuidades en la Puna de Jujuy (actual Argentina) durante la colonia. (G. E. Yocavil, Ed.) Población \& Sociedad, 25(1), 5-43.

Angiorama, C., V. Franco Salvi y A. Coronel. (2018b). Prácticas de labranza en el sur de Pozuelos (Puna de Jujuy, Argentina). Avances en el análisis de implementos agrícolas prehispánicos. Arqueología 25 (2): 33-50.

Angiorama, C., A. Coronel y M. Pigoni. (2018c). Agricultura a pequeña escala durante tiempos prehispánicos y coloniales en Chajarahuayco 25. (Puna de Jujuy, Argentina). Revista de Arqueología Histórica Argentina y Latinoamericana 12: 12- 36.

Angiorama, C.I, M.F. Becerra, A. Coronel, V. Franco Salvi, M. Giusta, M. S. Lauricella, M.J. Pérez Pieroni Y S. Rodríguez Curletto. (2019). Historia Ocupacional y prácticas productivas en Moreta (Puna de Jujuy, 
Argentina) durante tiempos prehispánicos y Coloniales. Relaciones de la Sociedad Argentina de Antropología XLIV (1): 13-34

Behrensmeyer, A.K. (1978). Taphonomic and ecological information from bone weathering. Palaeobiology 4: 150-162.

Carreras, J. (2020). Primeras aproximaciones al registro zooarqueológico de Pajchela Núcleo, Puna de Jujuy: un acercamiento a las prácticas de manejo y consumo de animales en un contexto agropastoril. Arqueología 26 (2): $13-31$

Erramouspe, V., Aschero, C., y Urquiza, S. (2017). Manejo de camélidos durante el Formativo temprano en la Puna Seca de Jujuy (Argentina). Intersecciones en Antropología (18): 295-303.

Gil Montero, R. (2004). Caravaneros y trashumantes en los Andes Meridionales. Población y familia indígena en la Puna de Jujuy 1770-1870. Lima, Perú: IEP.

Gil Montero, R. (2015). El mundo del trabajo indígena en los Andes durante la Colonial. Ensayo de opinión. Mundo de Antes n9: 13-42. ISSN 1514-982X/ ISSN en línea 2362-325X

Göbel, B. (2001). El ciclo anual de la producción pastoril en Huáncar (Jujuy, Argentina). En G. Mengoni Goñalons, D. Olivera y H. Yacobaccio (eds.) El uso de los camélidos a través del tiempo: 91-115. Grupo de Zooarqueología de Camélidos, Buenos Aires.

Grant, J. y Escola, P. (2015). La persistencia de un modo de producción doméstico durante el período Tardío: el caso de Corral Alto (Antofagasta de la Sierra, Argentina). Estudios Atacameños. Arqueología y antropología surandinas. vol. 51: 99 - 121. San Pedro de Atacama.

Grayson, D. K. (1984). Quantitative Zooarchaeology: Topics in the analysis of archaeological faunas. Academic Press, Orlando.

Hogg, A. G., P. Quan Hua, G. Blackwell, C. E. Buck, T. P. Guilderson, T. J. Heaton, M. Niu, J. G. Palmer, P. J. Reimer, R. W. Reimer, C. S. M. Turney, y S. R. H. Zimmerman. (2013). SHCal13 Southern Hemisphere calibration, 0-50,000 years cal. BP. Radiocarbon 55 (4): 1889-1903.

Izeta, A. (2008). Late Holocene camelid use tendencies in two diferent ecological zones of Northwestern Argentina. Quaternary International, 180(1): 135-144.

Kent, J. (1982). The domestication and exploitation of the South American camelids: methods of análisis and their application to circu-lacustrine archaeological sites in Bolivia and Peru. Phd Dissertation. St. Louis. Washington University.

Lema, C. (2006). Espacios de resistencia: el caso de Tebenquiche Chico. En Arqueología histórica en América Latina. Temas y discusiones recientes. P. Funari y F. Brittez (edit.), Ediciones Suárez. Mar del Plata.

Mengoni Goñalons, G. (1999). Cazadores de Guanacos de la estepa patagónica. Colección Tesis Doctorales. Sociedad Argentina de Antropología.

Madero, C. (2002). Modelo etnoarqueológico de estructura etaria en contextos pastoriles actuales de la puna de Jujuy. Cuadernos del Instituto Nacional de Antropología y Pensamiento Latinoamericano 19: 385-399.

Mengoni Goñalons, G. (2013). El aprovechamiento de la fauna en sociedades complejas del NOA: aspectos metodológicos y su aplicación a diferentes contextos arqueológicos. En: Williams, V. y B. Cremonte (eds.). "Al borde del Imperio: Paisajes sociales en áreas periféricas del Qollasuyu".

Mengoni Goñalons, G. L. y H. D. Yacobaccio (2006). The domestication of South American camelids: a view from the South-Central Andes. En: M. A. Zeder, D. Bradley, E. Emshwiller y B. D. Smith (eds.), documenting domestication: new genetic and archaeological paradigms, 228-244. University of California Press, Berkeley.

Mercolli, P. (2009a). Informe osteométrico efectuado en un esqueleto de llama procedente de Rinconada, Puna de Jujuy, Argentina. Ms.

Mercolli, P. (2009b) Informe osteométrico efectuado en un esqueleto de vicuña procedente de Rinconada, Puna de Jujuy, Argentina. Ms.

Mercolli, P. (2019). La importancia de los recursos silvestres y el manejo de los rebaños de llamas por parte de las sociedades humanas en la Quebrada de Humahuaca, Jujuy, Argentina (0-1536 d.C.). Tesis de Doctorado, Ms.

Mercolli, P. H. Mamani y D. Basso. (2018). Resultados del análisis de la muestra arqueofaunítica del sitio $\mathrm{COCH}-1.1$ (Cochinoca, Puna de Jujuy, Argentina). Cuadernos del INPL- Series Especiales. Vol 6 N²: 39-54.

Moreno, E. (2010). Arqueología de la caza de vicuñas en el área del salar de Antofalla, Puna de Atacama. Una aproximaion desde la arqueología del paisaje. Tesis doctoral. UCNyM- UNLP.

Moreno, E. Y C. Lema. (2012). El aprovechamiento de la vicuña en Tebenquiche chico, Siglos XVI y SXVII DC. Aportes científicos desde humanidades N9: 242-253. 
FH- UNC. ISSN: 1851-4464.

Nielsen, A. (2004). El Período de Desarrollos Regionales en los Andes Circumpuneños: aspectos cronológicos. Buenos Aires: ICA, FFyLL.

Nielsen, A. (2006a). Plazas para los antepasados: Descentralización y poder corporativo en las formaciones políticas preincaicas de los Andes circumpuneños. Estudios Atacameños, 31: 63- 89.

Nielsen, A. (2006b). Pobres Jefes: aspectos corporativos en las formaciones sociales pre-inkaicas de los Andes Circumpuneños. En C. y. Gnecco (Ed.), Contra la tiranía tipológica en arqueología: una visión desde Sudamérica: 121-150. Universidad de los Andes- CESO. Bogotá.

Olivera, D. (1997) La importancia del recurso Camelidae en la Puna de Atacama entre los 10.000 y 500 años AP. Estudios Atacameños 14: 29-41.

Olivera, D. Y J. Grant (2008). Economía y Ambiente durante el holoceno tardío (ca. 4500-400) de Antofagasta de la Sierra (Puna Meridional Argentina). En A. Acosta, D. Loponte y L. Mucciolo (eds.) Temas de Arqueología: Estudios tafonómicos y zooarqueológicos (1): 99-131.

Olivera, D. Y J. Grant. (2009). Puestos de altura de la puna argentina: Zooarqueología de Real Grande 1 y 6 y Alero Tomayoc. Revista del Museo de Antropología $N^{\circ}$ 2: 141-150. Córdoba.

Palomeque, S. (1995). Intercambios mercantiles y participación indígena en la 'Puna de Jujuy' a fines del período colonial. Andes 6:13-48.

Pérez Pieroni, M.J. (2013). Prácticas productivas y tradiciones tecnológicas: la manufactura cerámica prehispánica tardía y colonial en la cuenca sur de Pozuelos y el área de Santa Catalina, Puna de Jujuy, Argentina. Tesis de Doctorado. MS.

Pérez Pieroni, M.J. Y C.I. Angiorama. (2018). Evidencias arqueológicas de los siglos VII a XII AD en el asentamiento de Moreta (Puna de Jujuy, Argentina). Comechingonia 22(1): 185-210.

Puig, S. (1988). Craneología y craneometría de camélidos: diferenciación interespecífica y determinación de la edad. Xama 1: 43-56. Mendoza. Argentina.

Puig, S. Y Monge, S. (1983). Determinación de la edad en Lama guanicoe. Deserta 7: 246-270. Mendoza.

Puló, M. (1998). La vicuña: el oro que camina por los andes. Andes. Antropología e historia 9: 243-280.

Raffino, R. (2007 [1988]). Poblaciones indígenas en
Argentina: urbanismo y proceso social precolombino. Emecé, Buenos Aires.

Ratto, N. (2003). Estrategias de caza y propiedades del registro arqueológico en la Puna de Chaschuil (Departamento de Tinogasta, Catamarca, Argentina). Tesis de doctorado. MS

Rodríguez Curletto, S. Y Angiorama, C. (2019). Arte Rupestre y Morfometría Geométrica. Las manifestaciones rupestres de camélidos en el sur de Pozuelos (Puna de Jujuy, Argentina), durante los Desarrollos Regionales (9001430 DC). Complutum, 30 (2): 415-443.

Ruiz, M. Y M. Albeck. (1997). El Fenómeno Pukara visto desde la Puna Jujeña. Estudios Atacameños 12: 83-95. Universidad Católica del Norte. San Pedro de Atacama.

Samec, C., M. Morales Y H. Yacobaccio. (2014). Exploring Human Subsistence Strategies and Environmental Change through Stable Isotopes in the Dry Puna of Argentina. International Journal of Osteoarchaeology 24: 1-15

Vaquer, J; L. Eguía Y J. Carreras. (2018). Primeras aproximaciones al conjunto zooarqueológico del recinto 1 de casas Quemadas (Cusi Cusi, Rinconada, Jujuy). Cuadernos del INPL- Series Especiales. Vol 6 N²: 55-70.

Wheeler, J. C. (1982). Aging Llamas and Alpacas by their Teeth. Llama World 1:12-17.

Wing, E. (1975). Hunting and Herding in the Peruvian Andes. En Clason, A. T. (ed.). Archaeozoological Studies: 302-308. Amsterdam, North Holland Publishing Company.

Yacobaccio H. (2007). Andean camelid herding in the South Andes: ethnoarchaeological models for archaeozoological research. Anthropozoologica 42 (2): 143-154.

Yacobaccio, H. (2010). Osteometría de llamas y sus consecuencias arqueológicas. En M. Gutiérrez, M. De Nigris, P. Fernández, M. Giardina, A. Gil, A. Izeta, G. Neme y $\mathrm{H}$. Yacobaccio (eds.) Zooarqueología a principios del siglo XXI. Aportes teóricos, metodológicos y casos de estudio, pp. 65-75. Del Espinillo, Buenos Aires.

Yacobaccio, H. (2012). Intercambio y caravanas de llamas en el Sur Andino (3000-1000 AP). Comechingonia 16: 31-51.

Yacobaccio, H. (2020). Comentario. Comechingonia. Revista de Arqueología. Vol. Esp. 24, n 1, pp. 89-91. Primer semestre de 2020. ISSN: 0326-7911.

Yacobaccio, Hugo. (2009). The Historical Relationship between People and the Vicuña. En The Vicuña. Theory 
and Practice of Community-Based Wildlife Management. I. Gordon (Edit.): 7-20. Townsville: Springer.

Yacobaccio, H., C. Madero, M. Malmierca Y Mc. Reigadas. (1997-1998). Caza, domesticación y pastoreo de camélidos en la Puna Argentina. Relaciones de la Sociedad Argentina de Antropología XXXII-XXIII: 389-418. ISSN 0325-2221. Buenos Aires.

Yacobaccio, H Y C. Madero (2001). Ethnoarchaeology of a pastoral settlement of the andean plateau: An investigation of archaeological scale. En L. Kuznar (ed.) Ethnoarchaeology of Andean South America, pp. 84-96. Internacional Monographs in Prehistory, Ethnoarchaeological Series 4, Ann Arbor.
Yacobaccio, H., L. Killian y B. Vilá. (2007). La explotación de la vicuña durante el período colonial (1535-1810). El negocio de los cueros y lanas de vicuña. Todo es Historia N 483: 16-21.

Yacobaccio, H.D, M.P.Catá, M.R. Morales, P. Solá, M.S. Alonso, M. Rosenbush, C. Vázquez, C.T. Samec, B.I. Oxman y M. Cáceres. (2011). El Uso de cuevas por parte de pastores andinos: el caso de Cueva Quispe (Susques, Puna de Jujuy). En: Arqueología de la Puna Argentina: Perspectivas actuales en el estudio de a diversidad cultural. Cap 3: 33-48. Archeopres.

Yacobaccio, H. D. Y B. Vilá. (2016). A model for llama (Lama glama Linnaeus, 1758) domestication in the southern Andes. Anthropozoologica51 (1): 5-13. 